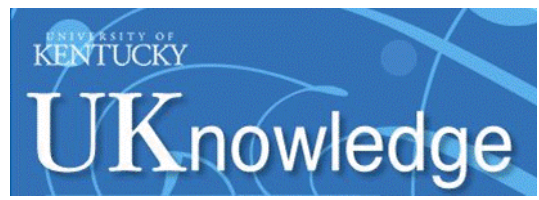

University of Kentucky

UKnowledge

Center for Computational Sciences Faculty

Publications

Center for Computational Sciences

3-30-2015

\title{
Band Gap Engineering via Doping: A Predictive Approach
}

Antonis N. Andriotis

Institute of Electronic Structure and Laser, Greece, andriot@iesl.forth.gr

Madhu Menon

University of Kentucky, super250@uky.edu

Follow this and additional works at: https://uknowledge.uky.edu/ccs_facpub

Part of the Computer Sciences Commons, and the Physics Commons

Right click to open a feedback form in a new tab to let us know how this document benefits you.

\section{Repository Citation}

Andriotis, Antonis N. and Menon, Madhu, "Band Gap Engineering via Doping: A Predictive Approach" (2015). Center for Computational Sciences Faculty Publications. 1.

https://uknowledge.uky.edu/ccs_facpub/1

This Article is brought to you for free and open access by the Center for Computational Sciences at UKnowledge. It has been accepted for inclusion in Center for Computational Sciences Faculty Publications by an authorized administrator of UKnowledge. For more information, please contact UKnowledge@lsv.uky.edu. 


\section{Band Gap Engineering via Doping: A Predictive Approach}

\section{Digital Object Identifier (DOI)}

http://dx.doi.org/10.1063/1.4916252

\section{Notes/Citation Information}

Published in Journal of Applied Physics, v. 117, no. 12, article 125708, p. 1-9.

Copyright 2015 American Institute of Physics. This article may be downloaded for personal use only. Any other use requires prior permission of the author and the American Institute of Physics.

The following article appeared in Journal of Applied Physics, v. 117, no. 12, article 125708, p. 1-9 and may be found at http://dx.doi.org/10.1063/1.4916252. 


\title{
Band gap engineering via doping: A predictive approach
}

\author{
Antonis N. Andriotis ${ }^{1, a)}$ and Madhu Menon ${ }^{2, b)}$ \\ ${ }^{1}$ Institute of Electronic Structure and Laser, FORTH, P.O. Box 1527, 71110 Heraklio, Crete, Greece \\ ${ }^{2}$ Department of Physics and Astronomy and Center for Computational Sciences, University of Kentucky, \\ Lexington, Kentucky 40506, USA
}

(Received 27 December 2014; accepted 16 March 2015; published online 30 March 2015)

\begin{abstract}
We employ an extension of Harrison's theory at the tight binding level of approximation to develop a predictive approach for band gap engineering involving isovalent doping of wide band gap semiconductors. Our results indicate that reasonably accurate predictions can be achieved at qualitative as well as quantitative levels. The predictive results were checked against ab initio ones obtained at the level of DFT/SGGA $+U$ approximation. The minor disagreements between predicted and $a b$ initio results can be attributed to the electronic processes not incorporated in Harrison's theory. These include processes such as the conduction band anticrossing [Shan et al., Phys. Rev. Lett. 82, 1221 (1999); Walukiewicz et al., Phys. Rev. Lett. 85, 1552 (2000)] and valence band anticrossing [Alberi et al., Phys. Rev. B 77, 073202 (2008); Appl. Phys. Lett. 92, 162105 (2008); Appl. Phys. Lett. 91, 051909 (2007); Phys. Rev. B 75, 045203 (2007)], as well as the multiorbital rehybridization. Another cause of disagreement between the results of our predictive approach and the $a b$ initio ones is shown to be the result of the shift of Fermi energy within the impurity band formed at the edge of the valence band maximum due to rehybridization. The validity of our approach is demonstrated with example applications for the systems $\mathrm{GaN}_{1-x} \mathrm{Sb}_{x}, \mathrm{GaP}_{1-x} \mathrm{Sb}_{x}, \mathrm{AlSb}_{1-x} \mathrm{P}_{x}, \mathrm{AlP}_{1-x} \mathrm{Sb}_{x}$, and $\mathrm{InP}_{1-x} \mathrm{Sb}_{x}$. C 2015 AIP Publishing LLC. [http://dx.doi.org/10.1063/1.4916252]
\end{abstract}

\section{INTRODUCTION}

Photoelectrochemical (PEC) water splitting is one of the most promising approaches for utilizing solar energy to produce clean fuels. ${ }^{7,8}$ However, the practical implementation of this technology is based on materials properties that are hardly met in nature. In particular, the basic criteria that these materials have to satisfy are the following: ${ }^{9}$ They have to (i) posses the correct band gap, (ii) straddle the redox potentials of the water reactions, and (iii) be stable against corrosion.

Band gap engineering has emerged as a powerful tool in the search for new materials that satisfy the criteria (i) and (ii) mentioned above. Of particular interest, PEC applications are materials harvesting the maximum possible sunlight energy. ${ }^{10,11}$ This makes wide band gap materials (such as GaN exhibiting a band gap of $3.45 \mathrm{eV}$ (Ref. 12)) very poor sunlight harvesters as they can absorb only $5 \%$ of the sunlight making use the UV range of the spectrum. Band engineering can assist in this direction and improves the absorbing power of such materials by reducing their band gap to values in the range of $1.5-2.1 \mathrm{eV}$, while while allowing them to satisfy the criteria (ii) and (iii) mentioned above. The band reduction will allow the materials to absorb in the visible range and their absorbing power to reach much higher levels.

In view of these, the logical first step in utilizing band gap engineering is to start with wide band gap materials (e.g., $\mathrm{TiO}_{2}, \mathrm{GaN}$, and $\mathrm{CdS}$ ) which, even though on their own cannot absorb more than $5 \%$ of the sunlight, can be made to

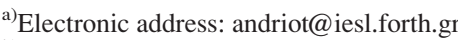

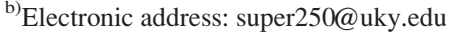

increase their absorbing power by employing suitable band gap engineering techniques. The simplest approach in this direction appears to be the doping (or alloying) of the wide band gap materials with the appropriate dopants which can turn these materials into narrower gap ones and, therefore, making them suitable for absorption in the visible range (absorbing approximately the 43\%) of the sunlight. In the following with the term doping, we will refer either to isovalent (alloying) or non-isovalent atom lattice substitution.

Upon doping a semiconductor, impurity states are created which could appear either within its gap or outside of it. If the impurity states are formed within the energy range of the energy gap, this will cause its reduction and most probably will shift the Fermi energy, $\mathrm{E}_{F}{ }^{13}$ into the impurity bands. On the other hand, if the impurity states are created outside the gap, it is expected that they will not affect the gap value in an appreciable way. The gap problem, thus, turns to be the problem of finding the appropriate dopant(s) which can reduce the energy gap of the wide band gap materials while complying with all the rest of the basic prerequisites that make them suitable for (direct) PEC water splitting (straddle the redox potentials of water, resist to corrosion).

The objective of the present work is to provide a simple theoretical screening approach of dopants which can indeed turn a wide band gap material into a potential candidate for efficient PEC water splitting (having a band gap in the range of $1.5-2.1 \mathrm{eV}$ as well as satisfying the other prerequisites). More specifically, our aim is to propose a predictive approach that allows for qualitative but realistic predictions for the effect of substitutional (anionic and/or cationic) doping on the energy gap which can be useful in the gap engineering investigations. 
In a recent report, we have demonstrated the efficacy of this approach for the case of Sb-doped GaN. In that work, the prediction that doping with a small concentration of $\mathrm{Sb}$ atoms can result in a substantial reduction in the energy gap of $\mathrm{GaN}$ to levels suitable for PEC water splitting was verified by experiments subsequently. ${ }^{14}$ This has provided the motivation for the present proposal of a predictive approach suitable for gap engineering. This is based on our recently proposed generalization of Harrison's tight binding (TB) theory ${ }^{15,16}$ for locating the valence band gap edge of doped semiconductors with respect to vacuum. In particular, we presented an extension of Harrison's theory applicable to ternaries and quaternaries within a virtual crystal type of approximation (VCA). ${ }^{13}$ This is a fundamental step in verifying whether the gap of a doped semiconductor straddles the redox potentials of water (i.e., it satisfies the (ii) of the prerequisites for PEC applications mentioned above). Furthermore, we used our proposed theory to justify that the doping of $\mathrm{GaN}$ with $\mathrm{Sb}$ can indeed lead to band gap narrowing in agreement with $a b$ initio and experimental results. ${ }^{14}$

In the present work, we investigate the predictive value and the limitations of our proposed theory by presenting applications on four additional new doped semiconductors with isovalent dopants and test the applicability of our theory by $a b$ initio calculations. We discuss the limitations of our approach and suggest possible improvements. It should be noted that our investigation of the effect of doping on the band gap narrowing is not merely restricted to the search for the appearance or not of impurity related gap states. It is argued that electron correlation effects not included in the VCA (our theory is based upon) affect the band gap as well. As discussed in Sec. IV, such processes include band repulsion, conduction band anticrossing (CBAC), ${ }^{1,2}$ and valence band anticrossing (VBAC) ${ }^{3-6}$ effects as well as the multiorbital rehybridization processes that can take place at energy locations close to the band edges.

In Sec. II, we include a brief review of our proposed extension of Harrison's theory. ${ }^{15,16}$ In Sec. III, we describe the computational details; while in Sec. IV we present the applications of our theory on four new systems, namely, $\mathrm{GaP}_{1-x} \mathrm{Sb}_{x}, \mathrm{AlSb}_{1-x} \mathrm{P}_{x}, \mathrm{AlP}_{1-x} \mathrm{Sb}_{x}$, and $\mathrm{InP}_{1-x} \mathrm{Sb}_{x}$. For completeness our results for $\mathrm{GaN}_{1-x} \mathrm{Sb}_{x}$ are also included. Finally, in Sec. V we accumulate the conclusions of our work.

\section{PROPOSED PREDICTIVE THEORY}

As stated in the above, our objective is to provide a simple theoretical tool for application in band gap engineering that is based on alloying/doping. Although our focus is mainly on III-V materials, the proposed model can be extended to IV and II-VI materials as well. Our approach is based on Harrison's tight binding (TB) model theory ${ }^{15,16}$ under the following assumptions which ensure the validity of the virtual crystal approximation:

(1) For small dopant concentrations, $x$, the valence band maximum $E_{V B M}^{\text {host }}$ of the host material does not change appreciably and the doped system can be sufficiently described within the virtual-crystal approximation.
(2) The energy position of the impurity (dopant) states is independent of the dopant concentration (even at the smallest possible concentrations). At higher concentrations, however, one can expect a broadening and even a small shifting of the dopant impurity states (due to interactions among the dopant atoms and/or changes in the bond lengths of the dopant bonds with the host atoms).

According to Harrison, ${ }^{15}$ for a heteropolar semiconductor $\mathrm{AB}$ ( $\mathrm{A}$ and $\mathrm{B}$ specifying the cations and anions, respectively), the valence band maximum (VBM), $\mathrm{E}_{V B M}^{A B}$, with respect to the vacuum, is approximately given by the following formula: ${ }^{15,16}$

$$
E_{V B M}^{A B}=\frac{\epsilon_{p}^{A}+\epsilon_{p}^{B}}{2}-\left\{\left(\frac{\epsilon_{p}^{A}-\epsilon_{p}^{B}}{2}\right)^{2}+\left(\frac{1.28 \hbar^{2}}{m d_{A B}^{2}}\right)^{2}\right\}^{1 / 2},
$$

where $m$ is the electron mass, $d_{A B}$ is the length of the bond $\mathrm{A}-\mathrm{B}$, and $\epsilon_{p}^{A}, \epsilon_{p}^{B}$ are the atomic $p$-electron orbital energies for the cations and anions, respectively.

Within the virtual crystal approximation, the above formalism can be generalized in a straightforward way for the case of the ternary $\mathrm{AB}_{1-x} \mathrm{C}_{x},(0 \leq x \leq 1)$ and the quaternary heteropolar semiconductors $\mathrm{D}_{y} \mathrm{~A}_{1-y} \mathrm{~B}_{1-x} \mathrm{C}_{x}, \quad(0 \leq x \leq 1$, $0 \leq y \leq 1)$, with $\mathrm{B}$ and $\mathrm{C}$ belonging to anion type, while $\mathrm{A}$ and $\mathrm{D}$ of cation type. Our generalization is based on the assumption that the codoped semiconductor $\mathrm{D}_{y} \mathrm{~A}_{1-y} \mathrm{~B}_{1-x} \mathrm{C}_{x}$ consists of virtual cations, $\bar{A}$, and virtual anions, $\bar{B}$, defined as follows:

$$
\bar{A}=y D+(1-y) A
$$

and

$$
\bar{B}=x C+(1-x) B .
$$

Thus, the VBM of the quaternary can be expressed as

$$
E_{V B M}^{\bar{A} \bar{B}}=\frac{\epsilon_{p}^{\bar{A}}+\epsilon_{p}^{\bar{B}}}{2}-\left\{\left(\frac{\epsilon_{p}^{\bar{A}}-\epsilon_{p}^{\bar{B}}}{2}\right)^{2}+\left(\frac{1.28 \hbar^{2}}{m d_{\bar{A} \bar{B}}^{2}}\right)^{2}\right\}^{1 / 2}
$$

where

$$
\begin{aligned}
\epsilon_{p}^{\bar{A}} & =(1-y) \epsilon_{p}^{A}+y \epsilon_{p}^{D}, \\
\epsilon_{p}^{\bar{B}} & =(1-x) \epsilon_{p}^{B}+x \epsilon_{p}^{C}
\end{aligned}
$$

and $d_{\bar{A} \bar{B}}$ is estimated by a straightforward generalization of Baranowski's approach (see Ref. 13, and references therein).

In the process of making a predictive approach, we look at the bonding and antibonding orbital energies not only between the atomic orbitals (AOs) but also between the host $s p^{3}$ hybrid orbitals (HOs) and the dopant-HOs or the dopant $s$ or $p$ AOs and check if these orbital energies lie within or outside the range of the band gap energy. The energies of bonds formed by the participation of $Y_{1}$ and $Y_{2}$ (atomic or hybrid) orbitals are then approximately estimated from the following formula: 


$$
E_{\text {bond }}^{Y_{1} Y_{2}}=\frac{\epsilon_{Y_{1}}+\epsilon_{Y_{2}}}{2} \pm\left\{\left(\frac{\epsilon_{Y_{1}}-\epsilon_{Y_{2}}}{2}\right)^{2}+V_{1}^{2}\right\}^{1 / 2}
$$

where $\epsilon_{Y_{1}}, \epsilon_{Y_{2}}$ are the energies of the $Y_{1}$ and $Y_{2}$ orbitals and $V_{1}$ their interaction coupling energy, the latter approximated (in $\mathrm{eV}$ ) by the expression

$$
V_{1}=\frac{7.62 \eta}{d^{2}}
$$

where $d$ is the bond length given in $\AA$ and $\eta$ is taken equal to $=3.78$ and 3.04 for the s- and p-orbitals, respectively. ${ }^{15}$ If the $Y_{i}$ orbital is taken to be a hybrid orbital then its energy $\epsilon_{Y_{i}}$ is the $s p^{3}$-hybrid energy, $\epsilon_{h, Y_{i}}$ obtained from Eq. (7) (Ref. 15)

$$
\epsilon_{h, Y_{i}}=\frac{\epsilon_{s, Y_{i}}+3 \epsilon_{p, Y_{i}}}{4} .
$$

If $Y_{i}$ is an $\mathrm{AO}$, then $\epsilon_{Y_{i}}$ is the corresponding AO-energy $(s$ or $p)$.

It is worth pointing out that our model approach has as starting elements the orbital energies of the species consisting the ternary/quaternary compounds. This starting point was used by Hjalmarson et al. ${ }^{17}$ in deriving a predictive approach for the dopant associated gap states in semiconductors. More precisely, the predictions of Hjalmarson et al. are based on the calculation of the so defined trap energy $E\left(V_{l}\right)$ which is based on the difference of the site diagonal orbital energies, $V_{l}$, between the free host and the free dopant atoms of the semiconductor compound corrected in terms of the $a b$ initio calculated electron DOS of the impurity cell of the doped semiconductor (see Eq. (1) of Ref. 17). The philosophy of our paper is different, however. In contrast to the approach of Hjalmarson et al., our method produces the trapenergy function making use of the $s p^{3}$ hybridization and the subsequent rehybridization due to the formation of bonding and antibonding states between dopant and the host hybrid orbitals. Thus, our approach attempts to reveal those factors underlying the creation of the impurity states. These ingredients are hidden in the electron DOS (Eq. (1)) of Ref. 17. Our proposed predictive theory attempts to circumvent the $a b$ initio calculations with the latter used only to check the predictions of our proposed simpler approach.

\section{COMPUTATIONAL DETAILS}

Equation (1) is used in order to locate the VBM energy, $E_{V B M}^{A B}$, of the host material $\mathrm{AB}$ with respect to vacuum. $\mathrm{AO}-$ energies and coupling parameters are taken from Harrison's Solid State Table ${ }^{15}$ as explained in Sec. II. Equations (4) and (7) are used to obtain $E_{V B M}^{\bar{A} \bar{B}}$ and energies of the bonding/antibonding orbitals, respectively, as a function of the orbital couplings. The conduction band minimum (CBM) is located at the energy $E_{V B M}^{A B}+E_{\text {gap }}^{A B}$, where $E_{\text {gap }}^{A B}$ is the energy gap of the host material AB obtained by ab initio calculations.

For our ab initio calculations, we use the DFT/GGA $+\mathrm{U}$ formalism $^{18}$ at the Perdew-Burke-Ernzerhof (PBE) ${ }^{19}$ level of approximation augmented by including Hubbard-U corrections based on Dudarev's approach ${ }^{20}$ as implemented in the Vienna $A b$-initio Simulation Package (VASP). ${ }^{21-23}$ The projected augmented wave (PAW) potential ${ }^{22,23}$ is used to describe the core electrons. After testing for convergence we settled for a $3 \times 6 \times 2 \Gamma$-centered pack for $\mathbf{k}$-vectors sampling. A kinetic energy cutoff of $550 \mathrm{eV}$ was found to be sufficient to achieve a total energy convergence of the energies of the systems to within $1 \mathrm{meV}$. Gaussian smearing of $0.05 \mathrm{eV}$ was chosen to accelerate the electronic convergence. The optimization of atomic positions (including full cell optimization) was allowed to proceed without any symmetry constraints until the force on each atom is less than $5 \mathrm{meV} / \AA$.

The $\mathrm{U}$ values used in the GGA $+\mathrm{U}$ formalism are: $\mathrm{U}_{p, A l}$ $=16.0 \mathrm{eV}, \mathrm{U}_{d, G a}=6.5 \mathrm{eV}, \mathrm{U}_{p, I n}=3.5 \mathrm{eV}, \mathrm{U}_{p, P}=-14.5 \mathrm{eV}$, and $\mathrm{U}_{p, N}=\mathrm{U}_{p, S b}=-4.2 \mathrm{eV}$. All other $\mathrm{U}$ values such as $\mathrm{U}_{p, G a}, \mathrm{U}_{s, G a}, \mathrm{U}_{s, N}$, and $\mathrm{U}_{s, S b}$ are set to zero. The U-values have been obtained by fitting to experimental and/or theoretical data for the band gap and, if available, the electron DOS (see Refs. 13 and 18). We use this GGA + U method to calculate the band structure and density of states (DOS). All self-consistent calculations were carried out using a convergence criterion of $10^{-6} \mathrm{eV}$.

The geometric structures in all cases were fully optimized without any symmetry constraints using the GGA + U method prior to performing calculations for band structure and DOS. We used large unit cells of 216 atoms in all cases in order to allow large dopant concentrations. In cases of systems with more than one dopant substitution, we have employed randomly chosen atomic configurations of the dopants within the unit cell.

We consider bulk GaN in the wurtzite structure, while zinc-blende structure was used for GaP, GaSb, AlP, AlSb, and InP.

\section{EXAMPLE APPLICATIONS}

\section{A. GaN doped with $\mathrm{Sb}$}

For the sake of completeness, we briefly present our previously reported results and extend our discussion on the effect of doping $\mathrm{GaN}$ by $\mathrm{Sb}$ with the latter substituting $\mathrm{N}$ sites in the GaN wurtzite lattice. ${ }^{13}$

$\mathrm{GaN}$ is a wide band gap material with a fundamental band gap of $\approx 3.4 \mathrm{eV} .{ }^{12,24}$ The experimental band gap of bulk $\mathrm{GaSb}$ is $0.73 \mathrm{eV} .{ }^{25}$ Using Eq. (7), in Fig. 1 we show the covalent bond energies (bonding and antibonding) between a Gahybrid orbital, $\mathrm{Ga}(\mathrm{HO})$, and an $s$ - or a $p$-AO or a hybrid-Sb orbital (to be denoted as $\mathrm{Sb}(\mathrm{s}), \mathrm{Sb}(\mathrm{p})$, or $\mathrm{Sb}(\mathrm{HO})$, respectively) as a function of the Ga-Sb bond length, $d=d_{G a-S b}$. As shown in Fig. 1, for $d_{G a-S b}$ in the range between the bond lengths $d_{G a N}$ and $d_{G a S b}$ of $\mathrm{GaN}$ and $\mathrm{GaSb}$, respectively, i.e., $d_{G a-S b}$ $\in\left[d_{G a N}, d_{G a S b}\right]$ and correspondingly for $V_{1}$ in the range $V_{1}$ $\in[3.5,6.5] \mathrm{eV}$, the $\mathrm{Ga}-\mathrm{Sb}$ bond energies for the most part lie between $E_{V B M}^{G a N}$ and $E_{C B M}^{G a N}$ for the bonding states of bonds formed between $\mathrm{Ga}(\mathrm{HO})-\mathrm{Sb}(\mathrm{HO})$ or $\mathrm{Ga}(\mathrm{HO})-\mathrm{Sb}(\mathrm{p})$ orbitals. This means that the impurity levels which are introduced by the $\mathrm{Sb}$-impurities can indeed shift $\mathrm{E}_{F}$ above the $E_{V B M}^{G a N}$ and lead, thus, to an appreciable reduction in the energy gap of GaN. ${ }^{13,14}$ The exact location of the Sb-impurity level depends on the Ga-Sb bond-length, $d_{G a-S b}$, that will be developed. For example, if we assume that $d_{G a-S b}=0.5\left(d_{G a N}+d_{G a S b}\right)$, then the Sb-impurity level can be located at approximately 


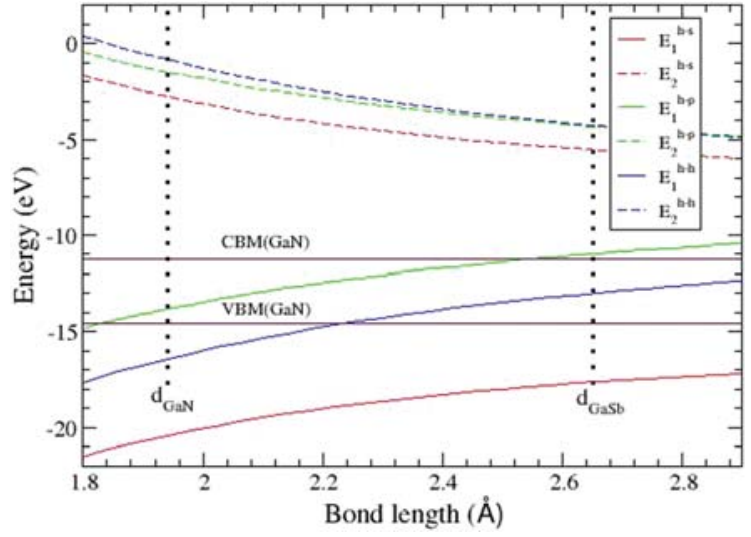

FIG. 1. $\mathrm{Ga}(\mathrm{Sb}) \mathrm{N}$ : Variation of the bonding and antibonding energies between Ga-HOs and Sb-HOs (denoted as $\mathrm{E}_{-}^{\mathrm{h}-\mathrm{h}}$ and $\mathrm{E}_{-}^{\mathrm{h}-\mathrm{h}}$ ) or between Ga-HOs and Sb-AOs (denoted as $\mathrm{E}_{1}^{\mathrm{h}-\mathrm{s}}, \mathrm{E}_{1}^{\mathrm{h}-\mathrm{p}}, \mathrm{E}_{2}^{\mathrm{h}-\mathrm{s}}$, and $\mathrm{E}_{2}^{\mathrm{h}-\mathrm{p}}$ ) as the Ga-Sb bond length is varied. The indices 1 and 2 refer to the bonding (antibonding) formations, respectively. Perpendicular dashed lines are drawn at bond length values corresponding to those for bulk $\mathrm{GaN}(1.94 \AA)$ and bulk $\mathrm{GaSb}(2.65 \AA)$.

$1.0-1.5 \mathrm{eV}$ above the $E_{V B M}^{G a N}$, and therefore, can reduce the energy gap of GaN by an appreciable amount. ${ }^{13,14}$ Our $a b$ initio results do confirm the above predictions. In particular, we find that even at a very small Sb-concentrations $\left(x_{S b} \leq 1 \%\right)$ impurity bands are formed within the energy gap of $\mathrm{GaN}$, "pinning" the Fermi energy leading not only to a significant reduction of the band gap value but also turning the direct nature of the GaN gap into an indirect one. ${ }^{13,14}$ The latter is due to the changes in the band dispersion as the Sb-concentration, $x_{S b}$, is increased. In particular, for $x_{S b}>6 \%$, the VBM is shifted away from the $\Gamma$-point while the CBM remains at the $\Gamma$-point. We cannot make a conclusive statement about these changes; they may be attributed to inter-impurity interactions and possibly to VAC processes. ${ }^{26}$ The change in the nature of the gap (direct to indirect) that we found and was observed in samples of high crystallinity, ${ }^{13,14}$ it was not found in amorphous $\mathrm{GaSb}_{x} \mathrm{~N}_{1-x}$ samples ${ }^{26}$ although the observed gap reduction in these samples was found in good agreement with our reported results. ${ }^{13,14}$

Our findings for the band gap engineering of $\mathrm{GaSb}_{x} \mathrm{~N}_{1-x}$ are also well supported by the work of Wei and Zunger, ${ }^{27}$ who studied the band bowing behavior of $\mathrm{GaN}_{x} \mathrm{As}_{1-x}$ in the full concentration range of $\mathrm{N}$-doped GaAs.

\section{B. GaP doped with Sb}

In the second example, we investigated the effect of substituting P-anions by $\mathrm{Sb}$ atoms in GaP. In Fig. 2, we present the variation of the energy positions of bonding and antibonding states resulting from the interaction of $\mathrm{Ga}(\mathrm{HO})$ and $\mathrm{Sb}(\mathrm{HO})$ or $\mathrm{Sb}(\mathrm{AO})$ as the $\mathrm{Ga}-\mathrm{Sb}$ interaction energy is varied according to the proposed theory in Sec. II. As seen in the figure, these level-energy variations fall outside the range of the gap energy for Ga-Sb bond lengths $d=d_{G a-S b}$ $\in\left[d_{G a P}, d_{G a S b}\right]$. These predictions are not changed if the coupling coefficient $\eta$ in Eq. (8) is reduced by $25 \%$.

From the presented behavior of the Sb-impurity states (resulting from the $\mathrm{GaP}$ doping by $\mathrm{Sb}$ substitutional dopants) it can be concluded that:

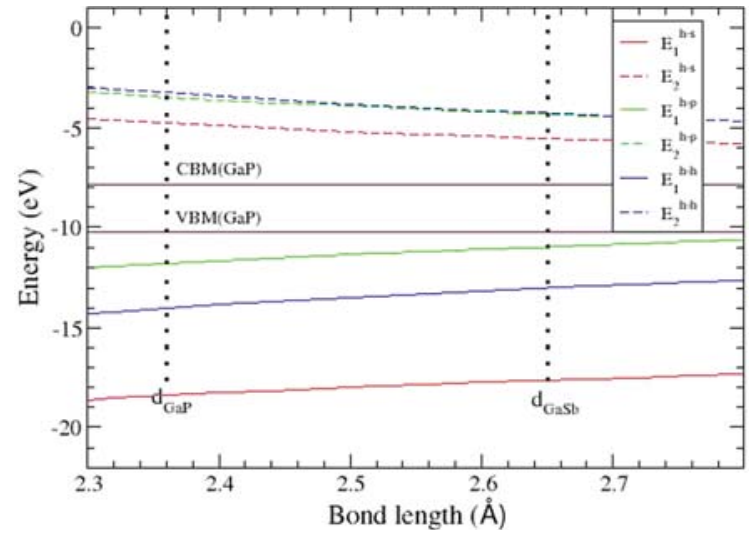

FIG. 2. As in Fig. 1 for $\mathrm{Ga}(\mathrm{Sb}) \mathrm{P}$. Bonding/antibonding states do not appear within the energy gap.

(1) The impurity states will be formed outside the gap region with having the $\mathrm{Ga}(\mathrm{HO})-\mathrm{Sb}(\mathrm{p})$ bonding states formed just below the VBM.

(2) There will be very little change in the energy gap due to the lack of the appearance of impurity related states in the gap.

According to our $a b$ initio results, the substitution of a $\mathrm{P}$ atom by a $\mathrm{Sb}$ atom and the subsequent relaxation of the crystal structure results in an increase in the volume of the unit cell and an increase in the Ga-Sb and the Ga-P bond-lengths as compared to that of the non-doped GaP. Specifically, the $\mathrm{Ga}-\mathrm{Sb}$ bond is approximately $10 \%$ greater than that of the $\mathrm{Ga}-\mathrm{P}$ of undoped material and is independent of the Sbconcentration. This increase makes the $\mathrm{Ga}-\mathrm{Sb}$ bonds of $\mathrm{GaSb}_{x} \mathrm{P}_{1-x}$ slightly greater than the average of the Ga-P and $\mathrm{Ga}-\mathrm{Sb}$ bonds of the undoped systems $\mathrm{GaP}$ and $\mathrm{GaSb}$, respectively. On the other hand, the Ga-P bond-lengths of the $\mathrm{Sb}$-doped systems are found to increase only slightly as the Sb-concentration, $x_{S b}$, increases and attains an increase of $0.5 \%$ (compared to the undoped case) for $x_{S b}=5 / 108$.

In contradistinction to the $\mathrm{GaSb}_{x} \mathrm{~N}_{1-x}$ case, the doping of $\mathrm{GaP}$ with $\mathrm{Sb}$ does not lead to the appearance of Sb-related states in the energy gap as shown in Fig. 2. This is justified by our ab initio results which do not predict any gap states. However, they do predict a small reduction of the GaP energy gap upon substitutional doping with $\mathrm{Sb}$ as shown in Fig. 3. In particular, we calculated the gap value of $\mathrm{GaSb}_{x} \mathrm{P}_{1-x}$ for Sb concentrations $x \in[0.0,7 / 108]$ in steps of 1/108. As shown in Fig. 3, the gap value exhibits a small overall drop of approximately $0.30 \mathrm{eV}$ from its $x=0$ value $(2.28 \mathrm{eV})$ in this range of Sb-concentrations. Our results for the energy gap of GaP are in excellent agreement with the experimental photoluminesence ones ${ }^{28}$ and other reported data. ${ }^{29}$ The gap is indirect, while differing significantly from the direct one ${ }^{32}$ and becoming direct upon Sb-doping even at the smallest Sb-concentrations $(\geq 1 / 108)$.

In order to get a qualitative understanding of the small gap reduction found by the $a b$ initio calculations, we investigated whether factors other than those captured by our VCA used in generalizing Harrison's TB theory (discussed in Sec. II) can account for this small discrepancy. With this in mind we first investigated the $a b$ initio obtained electron DOS of 


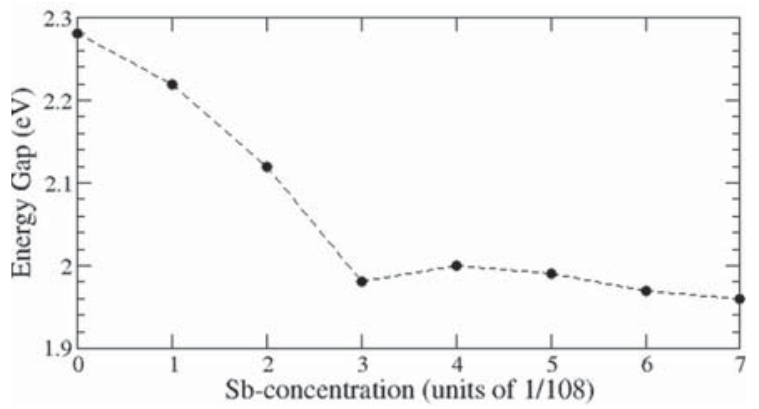

FIG. 3. $\mathrm{Ga}_{108} \mathrm{Sb}_{3} \mathrm{P}_{105}$ : Variation of the energy gap of $\mathrm{GaSb}_{x} \mathrm{P}_{1-x}$ as obtained by ab initio calculations.

$\mathrm{GaSb}_{x} \mathrm{P}_{1-x}$ at the gap edges. In Fig. 4, we show the electron DOS of $\mathrm{GaSb}_{x} \mathrm{P}_{1-x}$, for $x=3 / 108$. In particular, we present the total and the projected electron DOS over the host $(\mathrm{Ga}$ and $\mathrm{P}$ ) and impurity ( $\mathrm{Sb}$ ) atoms. It can be seen that both band edges include $\mathrm{Ga}, \mathrm{P}$, and $\mathrm{Sb}$ derived states outside the gap, thus confirming the results of our predictive approach of Sec. II and justifying the model Hamiltonian described below.

In view of the $a b$ initio obtained DOS results, we examine the possibility of the band edges being shifted as a result of the multiorbital coupling and rehybridization of $\mathrm{Ga}, \mathrm{Sb}$, and $\mathrm{P} p$-states. Such a rehybridization can be described by a $3 \times 3$ model Hamiltonian, $H_{\text {model }}$, which is constructed using the $p$-AO-energies $\epsilon_{p, k} ; \mathrm{k}=\mathrm{Ga}, \mathrm{P}, \mathrm{Sb}$ and the pair couplings $V_{p, i j} ; \mathrm{ij}=\mathrm{GaP}, \mathrm{GaSb}$, PSb between the $p$-AOs of these elements. ${ }^{15}$ In analogy with Eq. (1), the VBM can be obtained by solving (diagonalizing) $\left[H_{\text {model }}\right.$-E] according to

$$
H_{\text {model }}-E=\left(\begin{array}{ccc}
\epsilon_{p, G a}-E & V_{p, G a P} & V_{p, G a S b} \\
V_{p, G a P} & \epsilon_{p, P}-E & V_{p, P S b} \\
V_{p . G a S b} & V_{p, P S b} & \epsilon_{p, S b}-E
\end{array}\right)=0 .
$$

As shown in Fig. 5, the VBM (obtained from the solution of Eq. (10)) can shift upwards to a higher energy within the gap of $\mathrm{GaP}$ when the interaction parameters $V_{p, i j}$ are taken within the ranges dictated by Harrison's Solid State Table. ${ }^{15}$ The upward shift of the VBM is maximized when the $p$-AO energies of $\mathrm{Ga}, \mathrm{P}$, and $\mathrm{Sb}$ are taken into account and $V_{P S b}$ is
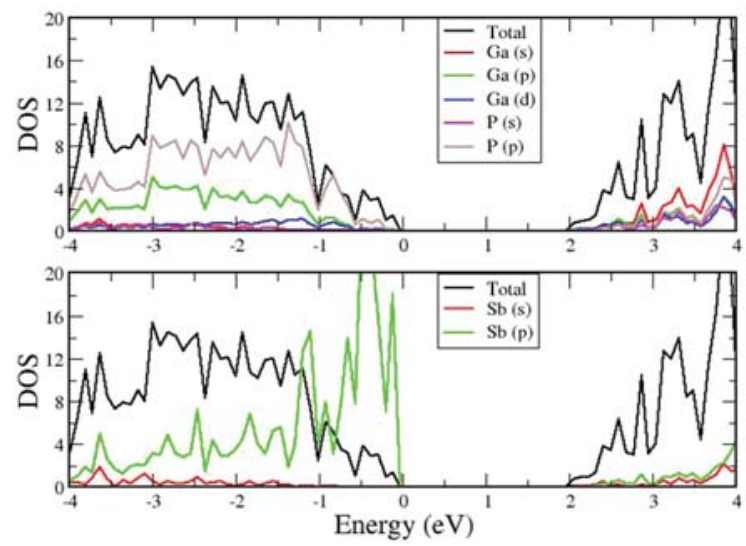

FIG. 4. $\mathrm{Ga}_{108} \mathrm{Sb}_{3} \mathrm{P}_{105}$ : Total and projected electron DOS on host and impurity atoms.

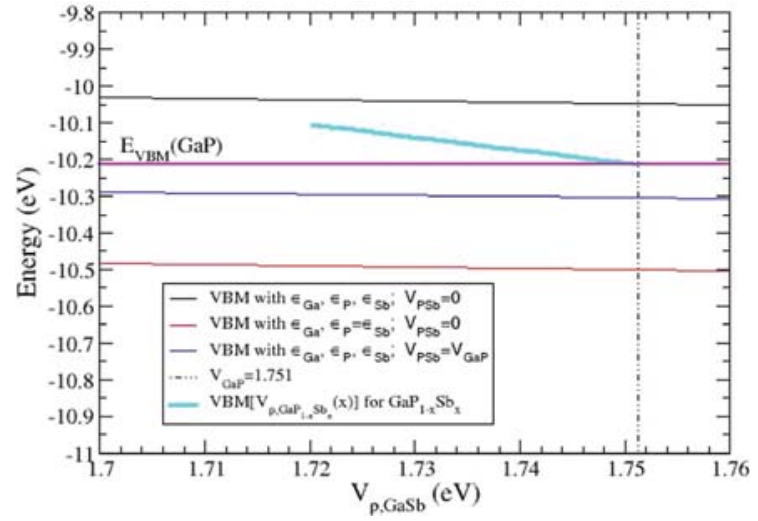

FIG. 5. Variation of the VBM with the interaction-coupling parameters $V_{p, i j}$, as obtained by diagonalizing the model Hamiltonian of Eq. (10). The cyan curve describes the variation of the VBM with the Sb-concentration (see Eqs. (11) and (12)).

smaller than $V_{G a P}$. This shift appears larger than the one we find when we use as anion energy the result of the virtual crystal approximation, i.e., if we use Eq. (4) (Ref. 13) assuming that

$$
d_{\bar{A} \bar{B}}=\left(1-x_{S b}\right) d_{G a P}+x_{S b} d_{G a S b}
$$

and

$$
V_{\bar{A} \bar{B}}=V_{p, G a P_{1-x_{S b}} S b_{x}}=\frac{1.28 \hbar^{2}}{m d_{\bar{A} \bar{B}}^{2}} .
$$

The model approximation dictated by Eq. (10) reduces to that of Eq. (4) in the limit $\epsilon_{p, P}=\epsilon_{p, S b}, V_{p, G a P}=V_{p, G a S b}$, and $V_{p, P S b}=0$.

The VBM shift obtained according to Eq. (10) correlates well with our ab initio results for the GaP-gap reduction upon Sb-doping. Equation (10) implies that a configuration interaction (CI) correlation term, which is partly inherent in the self-consistency of the DFT/SGGA $+U$ approach, can improve our TB extension of Harrison's model ${ }^{13}$ and allow us to attribute the observed reduction of the electron gap of $\mathrm{GaSb}_{x} \mathrm{P}_{1-x}$ to higher order correlation terms. It is worth noting that even with the approximate coupling interactions $V_{p, i j}$ that we have used, it is possible to obtain quantitative estimations of gap changes upon doping.

As is evident from our band structure calculations (see Fig. 6), upon Sb-doping, band repulsion, due to the appearance of an Sb-related band at the CBM-edge, leads to the lowering of the CBM-bands at the $\Gamma$-point resulting in the band gap changing from indirect into direct type. Furthermore, new $\mathrm{Sb}$ bands are introduced at the VBM in agreement with the predictions of our TB model Hamiltonian (Eq. (10)), which is based on the multiorbital mixing and rehybridization. These effects lead to the lifting of the band degeneracies at the VBM of the GaP-bands at the $\Gamma$-point causing a small reduction in the energy gap.

On the other hand, the band splittings observed at both the $\mathrm{CBM}$ and the VBM are found to become larger as the $\mathrm{Sb}$-concentration is increased. Accordingly, the significant Sb-related modifications of the energy bands of $\mathrm{GaSb}_{x} \mathrm{P}_{1-x}$ 

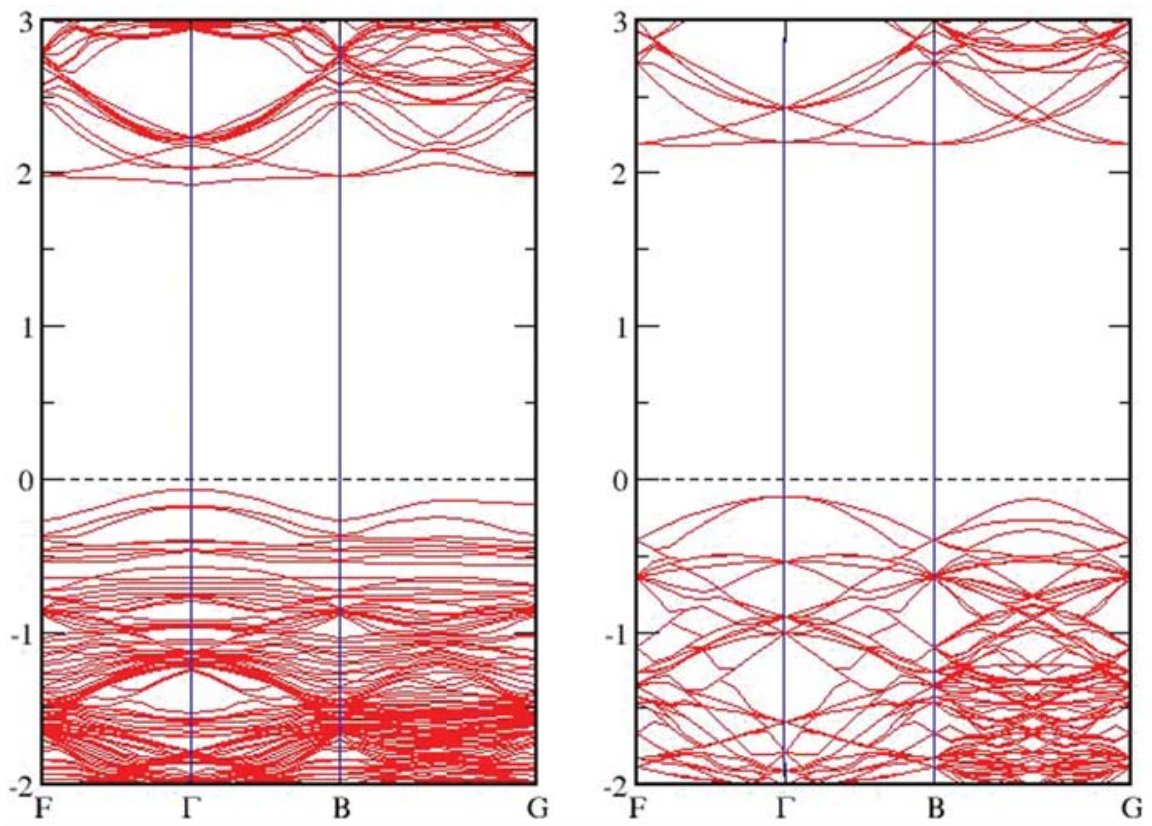

FIG. 6. Band structures for $\mathrm{Ga}_{108} \mathrm{Sb}_{3} \mathrm{P}_{105}$ (left panel) and for the impurity free GaP (right panel). The symmetry points (with an appropriate common multiplication factor) are as follows: $\mathrm{F}=(0.0,0.5,0.0)$, $\Gamma=(0.0,0.0,0.0), \mathrm{B}=(0.5,0.0,0.0)$, $\mathrm{G}=(0.0,0.0,0.5)$ could be interpreted within the framework of valence (and, respectively, conduction) band anticrossing [(VBAC), respectively, (CBAC) model $^{1-6}$ which is based on the strong coupling between the VBM (and, respectively, CBM) states and those of the dopant.

The similarity between the proposed model Hamiltonian (Eq. (10)) and the BAC (VBAC and CBAC) models is apparent with the former being equally well applicable for both the VBM edge and, if appropriately modified, for the CBM edge. CBAC (and, correspondingly, VBAC) effects are expected to be more pronounced in systems for which the electron affinity (and, correspondingly, the ionization energy) of the minority species (dopant) is smaller than that of the host. This is justified in the case of $\mathrm{GaSb}_{x} \mathrm{P}_{1-x}$ where the effect of Sb-doping was shown to be stronger on the VBM than on the CBM edge, while exhibiting strong dependence on Sb-concentration.

A systematic check of the dependence of our $a b$ initio results on the dopant-configuration is computationally prohibitive due to the large unit cell used. In fact, a complete structural optimization of all systems studied including configurational entropy contributions is computationally unfeasible and outside the scope of the present work. The results we present indicate mainly the trends found upon alloying and are sufficiently adequate for building up a predictive approach. Nevertheless, our results were checked for their dependence on the dopant-configuration in the case of $\mathrm{Ga}_{108} \mathrm{Sb}_{5} \mathrm{P}_{103}$. In particular, we obtained results for $\mathrm{Ga}_{108} \mathrm{Sb}_{5} \mathrm{P}_{103}$ in five different dopant-configurations (distributions of the 5-Sb dopants). These configurations covered cases of Sb-atoms placed far apart, forming nearest neighbors either on different P-planes or partially on the same P-plane. The total energies (per unit cell) of the systems studied were found to be within a range of $200 \mathrm{meV}$. Four of the dopant-configurations studied exhibit the lowest of the total energies and found with band-structure and DOS features almost identical to each other; their energy gap values exhibit a maximum variation of $5 \%$ of the value shown in Fig. 3. The fifth case was found to have the highest total energy and with the smallest gap value (approximately $10 \%$ smaller than the maximum found in the set of the other four cases).

\section{AISb doped with P and AIP doped with Sb}

As in Secs. IV A and IV B, we apply our theory to AlSb doped with $\mathrm{P}$ and to AlP doped by Sb. Thus, in Fig. 7 we present the variation of the energy positions of the bonding and antibonding states resulting from the interaction of $\mathrm{AlSb}(\mathrm{HO})$ and $\mathrm{P}(\mathrm{HO})$ or $\mathrm{P}(\mathrm{AO})$ as the $\mathrm{Al}-\mathrm{P}$ interaction (coupling) energy is varied. Similarly, in Fig. 8 we show the energetics of bonds formed from the interaction of $\mathrm{Al}(\mathrm{HO})$ with $\mathrm{Sb}(\mathrm{HO})$ or $\mathrm{Sb}(\mathrm{AO})$ as the $\mathrm{Al}-\mathrm{Sb}$ interaction is varied.

In both these cases, our model theory predicts that the doping introduces states with energies outside the range of the energy gap for bond length variations within the bond length values specified by the corresponding host materials (corresponding to dopant concentrations between $x=0$ and

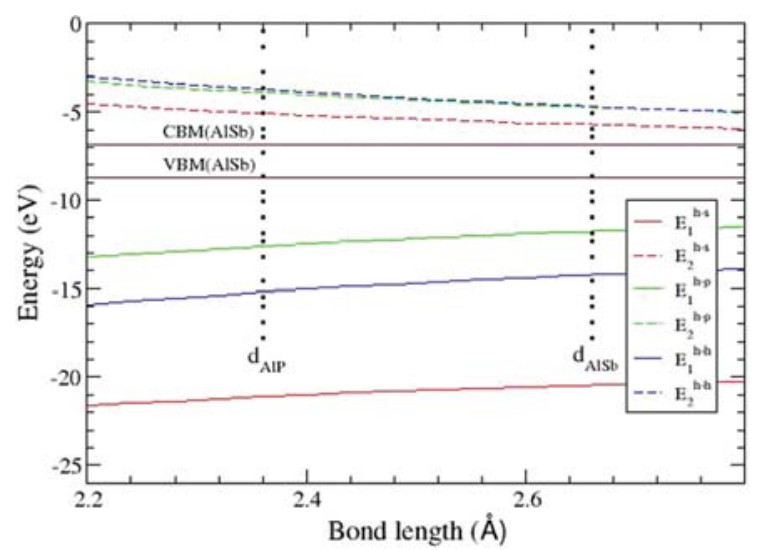

FIG. 7. As in Fig. 1 for $\mathrm{Al}(\mathrm{P}) \mathrm{Sb}$. 


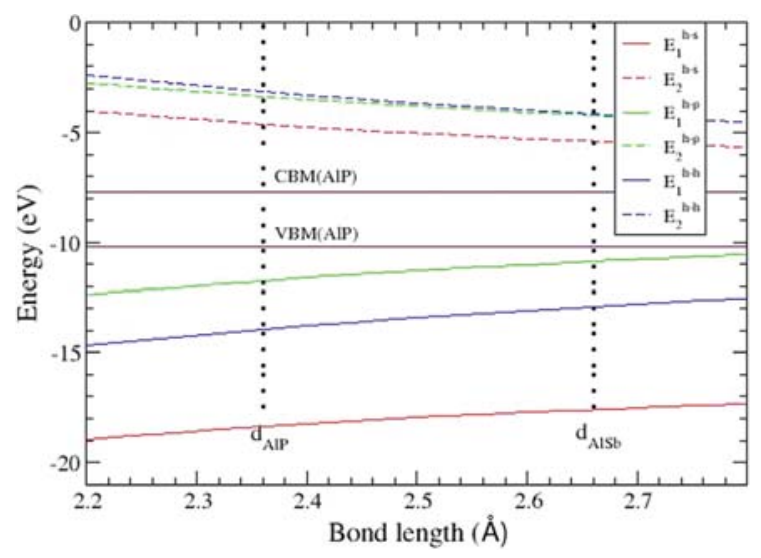

FIG. 8. As in Fig. 1 for $\mathrm{Al}(\mathrm{Sb}) \mathrm{P}$.

$x=1$ ). According to these results, one would not expect the doping in this range to affect the energy gap of the corresponding host materials appreciably. Once again, we find that our predictions are not changed if the coupling coefficient $\eta$ in Eq. (8) is reduced by $25 \%$.

An examination of the ab initio obtained band structure of $\mathrm{AlSb}_{x} \mathrm{P}_{1-x}$ for $x \in[0.0,7 / 108]$, however, shows the electronic gap of this material to narrow from $2.38 \mathrm{eV}$ (Ref. 33) to $1.67 \mathrm{eV}$ as the Sb-concentration, $x_{S b}$, increases from $x_{S b}=0.0$ to $x_{S b}=7 / 108$. This drop in the energy gap of $\mathrm{AlSb}_{x} \mathrm{P}_{1-x}$ is not nearly as rapid as the case for $\mathrm{GaSb}_{x} \mathrm{~N}_{1-x}$ as $x_{S b}$ is increased. Investigating further the disagreement between the $a b$ initio and predicted results, we look at the character of the electron DOS at the edges of the gap and compare the DOS and the band structure for $x_{S b}=0$ to that for $x_{S b} \neq 0.0$. It is observed that for $x_{S b}<1 \%$, a discrete Sb-related impurity band (of approximate bandwidth of $0.15 \mathrm{eV}$ ) is formed as part of the VB at the Fermi level at the edge of the VBM of undoped system; this is separated from the main part of the VB by a small gap of the order $0.15 \mathrm{eV}$. As $x_{S b}$ increases, the band width of the Sb-related impurity band increases reaching the value of $0.75 \mathrm{eV}$ for $x_{S b}=7 / 108$, while its separation from the rest of the VB is reduced to $0.07 \mathrm{eV}$. This is illustrated in the band structures of $\mathrm{Al}_{108} \mathrm{Sb}_{2} \mathrm{P}_{106}$ (Fig. 9, left) and $\mathrm{Al}_{108} \mathrm{Sb}_{7} \mathrm{P}_{101}$ (Fig. 9, right) where the broadening of the impurity band is evident upon increasing the $\mathrm{Sb}$ concentration. As a result, the Sb-related impurity band "pins" the Fermi level as in the case of the $\mathrm{GaN}_{1-x} \mathrm{Sb}_{x}$ case (see Sec. IV A). However, in $\mathrm{AlP}_{1-x} \mathrm{Sb}_{x}$ the Fermi level does not stabilize so quickly as it follows the increase in the band width of the impurity band. Instead, the Fermi energy change is more gradual for $x_{S b}$ $\leq 3 / 108$ and starts stabilizing only for $x_{S b} \geq 4 / 108$. Furthermore, it is found that the $d$-states of both $\mathrm{P}$ and $\mathrm{Al}$, which characterize the CBM, are shifted to lower energies in the presence of the $\mathrm{Sb}(d)$ states for $x_{S b} \neq 0.0$ resulting, thus, to gap value changes. We attribute this mainly to the rehybridization of the host $d$-states in the presence of the $\mathrm{Sb}$-ones, a factor which is not taken into account by our virtual crystal generalization of Harrison's theory. In principal, this effect could be taken into account by our model approximation described by Eq. (10). However, the lack of accurate coupling orbital interactions does not allow us to make accurate quantitative predictions. Analogous observations about the effect of the $d$-states have been reported in the case of $\mathrm{GaN}_{1-x} \mathrm{P}_{x}$.

It is worthwhile to further investigate if the band repulsion that is observed at the CBM can be associated with multiorbital mixing and/or CBAC effects. ${ }^{1,2}$ The latter can be justified as due to the smaller electron affinity of the host anions $(\mathrm{P})$ as compared to that of the dopant $(\mathrm{Sb})$.

On the other hand, our $a b$ initio calculations show that the energy gap of AlSb does not change upon doping with $\mathrm{P}$. The gap is found to be $0.75 \mathrm{eV}$ for P-concentrations $x_{P} \in[0.00,7 / 108]$. Although the gap value we find is less than the half of the experimental value $(1.615 \mathrm{eV}-$ indirect $^{31}$ ), however its non variation with $x_{P}$ is in agreement with our prediction and supports our predictive approach.
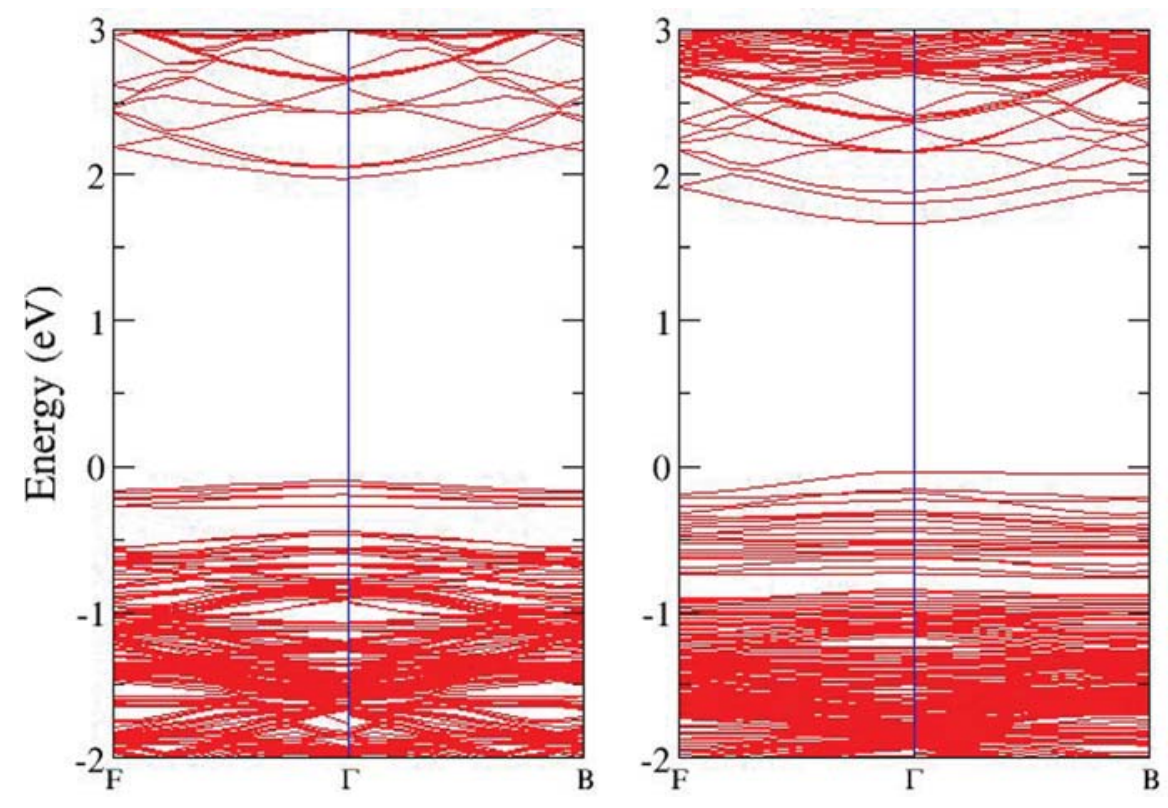

FIG. 9. Ab initio calculated band structures for $\mathrm{Al}_{108} \mathrm{Sb}_{2} \mathrm{P}_{106}$ (left) and $\mathrm{Al}_{108} \mathrm{Sb}_{7} \mathrm{P}_{101}$ (right). The Fermi level appears at the top of the impurity band which is formed at the VBM (of AlP) upon $\mathrm{Sb}$ substitution; it becomes broader as $\mathrm{Sb}$ concentration increases as shown on right. 


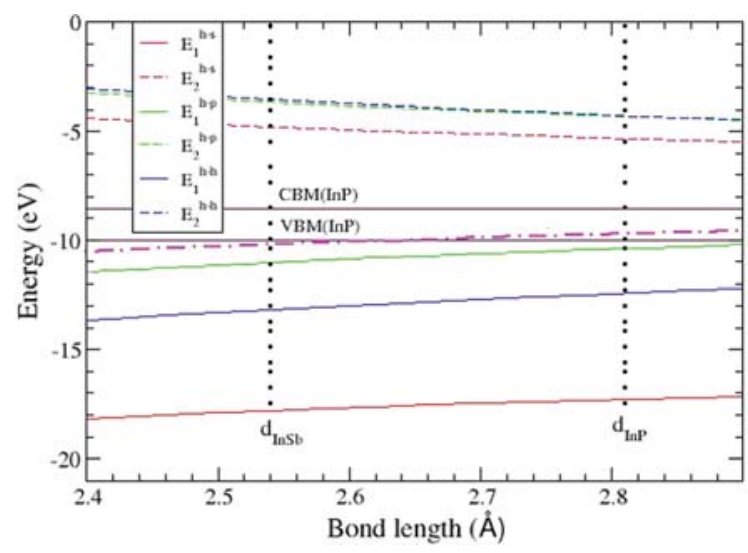

FIG. 10. As in Fig. 1 for $\operatorname{In}(\mathrm{Sb}) \mathrm{P}$. The dashed-dotted curve corresponds to $\mathrm{E}_{1}^{\mathrm{h}-\mathrm{p}}$ hybrid energy if the coupling coefficient $\eta$ in Eq. (8) is reduced by $25 \%$.

\section{InP doped with Sb}

In the case of $\mathrm{InP}_{1-x} \mathrm{Sb}_{x}$, our ab initio calculations indicate that the direct energy gap of the undoped InP (1.42-1.47 eV (Ref. 29)) drops upon Sb-doping, reaching the value of $1.08 \mathrm{eV}$ for Sb-concentration $x=6 / 108$. In Fig. 10, it can be seen that according to our predictive approach no substantial change in the energy gap of InP is expected upon Sb-doping. However, contrary to the previous cases, we see that hybrids involving the $\mathrm{Sb}(p)$ AOs can form bonding hybrids near the VBM if the In-Sb coupling constant is changed slightly. In particular, a small change in the coupling interaction could be adequate to shift the energy of these bonding hybrids within the energy gap and lead to a reduction in the energy gap. This is confirmed by our predictive approach. That is, by reducing the coupling coefficient $\eta$ in Eq. (8) by $25 \%$ (i.e., from 3.04 to 2.34), we find that the $E_{1}^{\mathrm{h}-\mathrm{p}}$ energies merge into the energy gap (dashed-dotted curve in Fig. 10) and therefore can lead to the gap reduction.
This, in fact, is justified by our ab initio calculations as shown in Fig. 11 where the electron DOS and the band structure of $\mathrm{InP}_{1-x} \mathrm{Sb}_{x}$ for various $\mathrm{Sb}$-concentrations are presented. As can be seen in the figure, the $\mathrm{Sb}(p)$-related states appear at the edge of the VBM; they form a hybridized Sbrelated band exhibiting a band width which increases as the $\mathrm{Sb}$-concentration increases. For Sb-concentration smaller than $10 \%$, the band width can extend up to $1.0 \mathrm{eV}$. Since the ionization energy of the dopant $(\mathrm{Sb})$ is much smaller than that of the host anions $(\mathrm{P})$, the band modifications observed at the edge of the VB could be attributed to VBAC effects. ${ }^{3-6}$ All these effects can well justify the reduction in the InP gap upon Sb-doping.

\section{CONCLUSION}

The example applications presented in Secs. IV A-IV D demonstrate that gap engineering predictions based on isovalent alloying of wide band gap semiconductors can be made at a qualitative and even a quantitative level. This has been achieved using extensions of Harrison's theory ${ }^{13,15}$ at the tight binding level and the virtual crystal type of approximation. In order to reveal the electronic processes underlying band-gap changes upon doping, instead of relying directly on the free atomic orbital energies as used previously (e.g., Refs. 15-17), our predictive approach is based on the energetics of the bonding and antibonding orbitals (that can be formed upon doping) in relation to the energy range of the energy gap. Some of our predictive results were found to be in excellent agreement with corresponding $a b$ initio ones obtained at the level of DFT/SGGA + U approximation. It has been shown that minor disagreements between predicted and ab initio results can be attributed to electronic processes not incorporated in Harrison's extended theory within the VCA. In particular, they are attributed to electron correlation effects which cannot be captured at the level of the virtual crystal type of approximation upon which our extension is
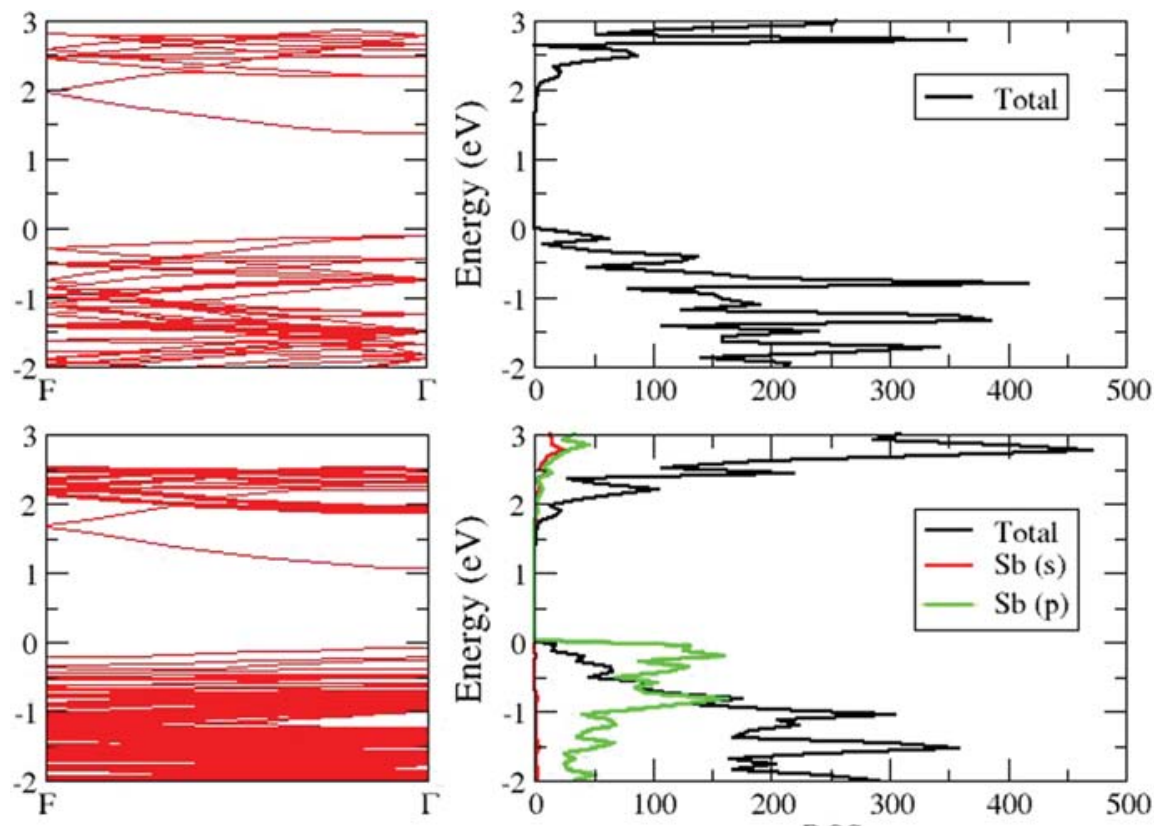

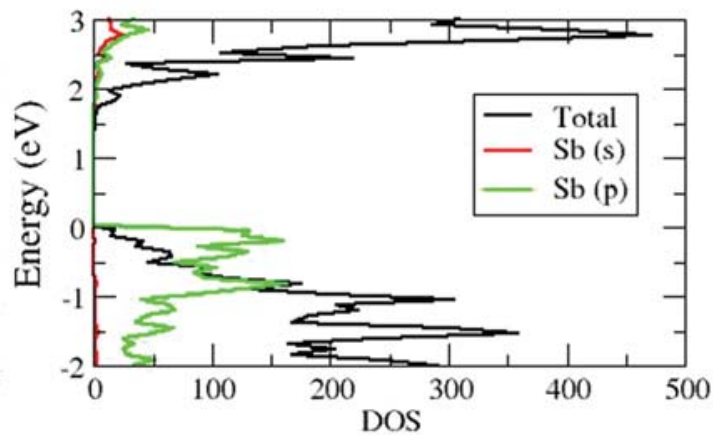

FIG. 11. Ab initio calculated electron DOS and band structures for $\operatorname{In}_{108} \mathrm{P}_{108}$ (top) and $\mathrm{In}_{108} \mathrm{Sb}_{4} \mathrm{P}_{104}$ (bottom). Upon increasing the Sb-concentration, the Sb-related band, appearing at the VBM, gets wider leading to the decrease of the electron gap. 
based. Such effects include (among others) band repulsion as well as the $\mathrm{CBAC}^{1,2}$ and $\mathrm{VBAC}^{3-6}$ processes and the multiorbital rehybridization. The latter, as was shown, results from the simultaneous presence of AOs of all the constituent atoms at either the edge of VBM or at that of the CBM. This rehybridization introduces a CI-type of interaction which has the effect of shifting the VBM (and, correspondingly, the $\mathrm{CBM}$ ) to higher (and, correspondingly, to lower) energies than that obtained by generalizing Harrison's theory within the virtual crystal type of approximation. As demonstrated in the present work the multiorbital rehybridization can account for the changes in the VBM in a satisfactory way. The changes in the CBM can also be equally well obtained by appropriately modifying Eq. (10). Another cause of disagreement between the results of our predictive approach and the $a b$ initio ones is attributed to the shift of the Fermi energy within the impurity band at the edge of the VBM as a result of rehybridization. By including all these major contributions into the proposed extension of Harrison's theory its predictive power can be improved by attaining satisfactory quantitative accuracy.

The proposed predictive approach can be used as a simple and efficient tool in the screening approaches designed to find the gap behavior of possible synthetically produced ternary and quaternary materials that can be formed by doping wide band gap materials.

\section{ACKNOWLEDGMENTS}

M.M. acknowledges funding support from NSF SOLAR project (DMS 1125909) and Kentucky Science and Engineering Foundation (KSEF-13-RDE-017). The support from NSF Award (IIA-1355438) was used to develop the computational techniques.

${ }^{1}$ W. Shan, W. Walukiewicz, J. W. Ager III, E. E. Haller, J. F. Geisz, D. J. Friedman, J. M. Olson, and S. R. Kurtz, Phys. Rev. Lett. 82, 1221 (1999).

${ }^{2}$ W. Walukiewicz, W. Shan, K. M. Yu, J. W. Ager, E. E. Haller, I. Miotkowski, M. J. Seong, H. Alawadhi, and A. K. Ramdas, Phys. Rev. Lett. 85, 1552 (2000).

${ }^{3}$ K. Alberi, J. Blacksberg, L. D. Bell, S. Nikzad, K. M. Yu, O. D. Dubon, and W. Walukiewicz, Phys. Rev. B 77, 073202 (2008).

${ }^{4}$ K. Alberi, O. D. Dubon, W. Walukiewicz, K. M. Yu, J. A. Gupta, and J.M. Baribeau, Appl. Phys. Lett. 92, 162105 (2008).
${ }^{5}$ K. Alberi, O. D. Dubon, W. Walukiewicz, K. M. Yu, K. Bertulis, and A. Krotkus, Appl. Phys. Lett. 91, 051909 (2007).

${ }^{6}$ K. Alberi, J. Wu, W. Walukiewicz, K. M. Yu, O. D. Dubon, S. P. Watkins, C. X. Wang, X. Liu, Y. J. Cho, and J. Furdyna, Phys. Rev. B 75, 045203 (2007).

${ }^{7}$ N. S. Lewis and D. G. Nocera, Proc. Natl. Acad. Sci. 103, 15729 (2006).

${ }^{8}$ A. J. Bard and M. A. Fox, Acc. Chem. Res. 28, 141 (1995).

${ }^{9}$ Z. Chen, T. F. Jaramillo, T. G. Deutsch, A. Kleiman-Shwarsctein, A. J. Forman, and N. Gaillard, J. Mater. Res. 25, 3 (2010).

${ }^{10}$ S. G. Kumar and L. G. Devi, J. Phys. Chem. A 115, 13211 (2011).

${ }^{11}$ K. Maeda and K. Domen, J. Phys. Chem. Lett. 1, 2655 (2010).

${ }^{12}$ B. Monemar, Phys. Rev. B 10, 676 (1974).

${ }^{13}$ A. N. Andriotis, R. M. Sheetz, E. Richter, and M. Menon, J. Phys.: Condens. Matter 26, 055013 (2014).

${ }^{14}$ S. Sunkara, V. K. Vendra, J. Jasinski, T. Deutsch, A. N. Andriotis, M. Menon, and M. Sunkara, "New Visible Light Absorbing Materials for Solar Fuels, $\mathrm{Ga}\left(\mathrm{Sb}_{x}\right) \mathrm{N}_{1-x}$," Adv. Mater. (published online).

${ }^{15}$ W. A. Harrison, Elementary Electronic Structure (World Scientific, 1999).

${ }^{16}$ W. A. Harrison and J. Tersoff, J. Vac. Sci. Technol., B 4, 1068 (1986).

${ }^{17}$ H. P. Hjalmarson, P. Vogl, D. J. Wolford, and J. D. Dow, Phys. Rev. Lett. 44, 810 (1980)

${ }^{18}$ A. N. Andriotis, G. Mpourmpakis, S. Lisenkov, R. M. Sheetz, and M. Menon, Phys. Status Solidi B 250, 356 (2013).

${ }^{19}$ J. P. Perdew, K. Burke, and M. Ernzerhof, Phys. Rev. Lett. 77, 3865 (1996).

${ }^{20}$ S. L. Dudarev, G. A. Botton, S. Y. Savrasov, C. J. Humphreys, and A. P. Sutton, Phys. Rev. B 57, 1505 (1998).

${ }^{21}$ G. Kresse and J. Hafner, Phys. Rev. B 47, 558 (1993).

${ }^{22}$ G. Kresse and D. Joubert, Phys. Rev. B 59, 1758 (1999).

${ }^{23}$ P. E. Blöchl, Phys. Rev. B 50, 17953 (1994).

${ }^{24}$ I. Vurgaftman and J. R. Meyer, J. Appl. Phys. 94, 3675 (2003).

${ }^{25}$ A. Chandola, R. Pino, and P. S. Dutta, Semicond. Sci. Technol. 20, 886 (2005).

${ }^{26}$ K. Yu, W. Sarney, S. Novikov, D. Detert, R. Zhao, J. Denlinger, S. Svensson, O. Dubon, W. Walukiewicz, and C. T. Foxon, Appl. Phys. Lett. 102, 102104 (2013).

${ }^{27}$ S. H. Wei and A. Zunger, Phys. Rev. Lett. 76, 664 (1996).

${ }^{28}$ W. Shan, W. Walukiewicz, K. M. Yu, J. Wu, J. W. I. Ager, E. E. Haller, H. P. Xin, and C. W. Tu, Appl. Phys. Lett. 76, 3251 (2000).

${ }^{29}$ I. Vurgaftman, J. R. Meyer, and L. R. Ram-Mohan, J. Appl. Phys. 89, 5815 (2001).

${ }^{30}$ R. Cherian and P. Mahadevan, Phys. Rev. B 76, 075205 (2007).

${ }^{31}$ A. Gruneis, G. Kresse, Y. Hinuma, and F. Oba, Phy. Rev. Lett. 112, 096401 (2014).

${ }^{32}$ The small value of the direct gap ( $2.31 \mathrm{eV}$ shown in Fig. 3), is due to zone folding effects. In the unfolded zone scheme, this is found to be much larger $(4.03 \mathrm{eV})$. The experimental value of the direct gap $(\approx 2.78 \mathrm{eV})$ may be better approximated using different set of U-values. However, U-values other than the ones we have used in this work deteriorate significantly the indirect gap value.

${ }^{33}$ This in excellent agreement with reported values for the indirect gap of AlSb spanning the range [2.35-2.39] eV (Ref. 29). 